EA 4272

\title{
Le contrôle de gestion des services : réflexion sur les fondements et l'instrumentation
}

\section{François Meyssonnier*}

\author{
2012/17
}

\section{*LEMNA - Université de Nantes}

Laboratoire d'Economie et de Management Nantes-Atlantique

Université de Nantes

INSTITUT

d'ECONOMIE

et de MANAGEMENT

de NANTES - IAE
Chemin de la Censive du Tertre - BP 52231

44322 Nantes cedex 3 - France

www.univ-nantes.fr/iemn-iae/recherche

Tél. +33 (0)2 40141717 - Fax +33 (0)2 40141749 


\title{
LE CONTROLE DE GESTION DES SERVICES REFLEXION SUR LES FONDEMENTS ET L'INSTRUMENTATION
}

Service industry management control: a reflexion about its essence and tools

\author{
François MEYSSONNIER \\ Professeur des Universités \\ Institut d'Economie et de Management de Nantes - IAE \\ LEMNA (Université de Nantes) \\ Courriel : francois.meyssonnier@univ-nantes.fr
}

\begin{abstract}
Résumé :
Les caractéristiques des activités de service sont étudiées et un cadre conceptuel unifié de référence est développé.

Les préoccupations principales du contrôle de gestion des services qui en découlent ainsi que les instrumentations associées sont présentées : l'évaluation de l'efficience des unités d'un réseau, la standardisation des processus productifs, la gestion des temps et des délais, la gestion des revenus et des capacités, la mesure de la qualité de service, la mise sous tension de l'entreprise de service.
\end{abstract}

Des recherches récentes sur le contrôle dans les services sont alors mobilisées afin de comprendre comment les pratiques et outils précédemment identifiés s'intègrent aux systèmes globaux de pilotage de la performance dans les entreprises de service.

Mots Clés : contrôle de gestion des services, objectifs du contrôle, outils du contrôle, systèmes de pilotage de la performance.

\begin{abstract}
$\underline{\text { Abstract: }}$
Characteristics of service activities are reviewed and a unified conceptual framework of reference is developed.

The main considerations of service industry management control derived from it, as well as associated practices and tools, are discussed: efficiency assessment of network units, standardization of production processes, delivery and waiting time management, yield and capacity management, service quality measurement, organization stretch of a service company.

Recent researches regarding management in service activities are then considered to understand how previously identified practices and tools are integrated in global performance management systems of companies dedicated to those activities.

Key Words: service industry management control, service industry control goals, service industry control tools, performance management systems.
\end{abstract}




\section{Introduction}

Les économies développées sont de plus en plus composées d'activités de service. En France aujourd'hui seulement un quart des emplois se trouve dans l'agriculture, l'industrie et la construction réunies. Les services administrés représentent un gros quart des emplois totaux et les services marchands une petite moitié. Notre économie est donc aujourd'hui fondamentalement une économie de service.

La structure de l'économie a changé mais les modes de gestion ont été façonnés dans le contexte d'une économie encore dominée par sa composante industrielle. Ainsi, les outils et la fonction de contrôle de gestion ont été essentiellement développés au tournant du $20^{\text {ème }}$ siècle aux Etats-Unis pour rationaliser les méthodes de gestion et accroître l'efficience des grandes entreprises industrielles. Les auteurs de référence de cette époque sont bien connus : Taylor (organisation scientifique du travail), Brown (pilotage de la performance financière), Wilson (optimisation logistique), Sloan (administration et budgets), etc. Les grandes entreprises pionnières étaient essentiellement des firmes industrielles (de la chimie, de la métallurgie ou de l'automobile) même si les compagnies ferroviaires ont joué un rôle non négligeable. Si on trouve parfois dans les services la source de certains outils (le taux de rotation des ventes dans la grande distribution étant à l'origine de la décomposition classique du ROI par exemple), les méthodes du contrôle de gestion sont profondément imprégnées des problématiques de la transformation matérielle des biens : la standardisation des techniques et des process de production, la volonté d'homogénéiser la production de masse et de réaliser des économies d'échelle, la gestion et le contrôle des stocks, etc.

Certes une seconde vague majeure d'innovations en gestion des opérations et en contrôle opérationnel est venue du Japon (elle a été connue par le monde dans les années 80) avec le grand mouvement de qualité totale en gestion de production, les méthodes d'amélioration continue (kaizen costing) et de coût cible (target costing) pour la maîtrise des coûts, le kanban et le zéro stock en fabrication, le zéro délai en logistique. Elle a remis en cause l'optimisation locale de la production de biens pour favoriser les interrelations sur l'ensemble des processus de la chaîne de valeur, les flux tendus tirés par l'aval et la réduction des stocks tampons. La valeur fournie au client, qu'il soit interne ou externe, était au cœur de cette révolution conceptuelle qui rapprochait l'industrie des préoccupations des entreprises de service. Toutefois les activités de service se caractérisent par des différences fortes avec les activités industrielles dans quatre caractéristiques fondamentales, reprises dans le fameux acronyme IHIP (objet d'une synthèse de la littérature sur le sujet réalisée par Zethaml et alii en 1985) qui est présent dans tous les manuels ou ouvrages consacrés au management des services depuis les années 80 : le caractère immatériel (Intangibility); la faible standardisation (Heterogeneity) ; la production et la consommation simultanées (Inseparability); le stockage impossible (Perishability).

Par ailleurs, on peut distinguer deux aspects principaux dans le contrôle de gestion : la mesure de la performance financière globale (contrôle des résultats) et l'amélioration de la qualité des gestions locales (aide à la décision et contrôle des comportements). Dans les business schools nord-américaines les enseignements de contrôle de gestion sont souvent scindés et rattachés pour partie à la comptabilité (calcul et analyse des coûts, contrôle budgétaire) et pour partie à la gestion des opérations (méthodes d'action sur les coûts, la qualité et les délais ; indicateurs et tableaux de bord). Dans les entreprises, on constate aussi une différenciation des fonctions de contrôle de gestion: plutôt tournées vers la modélisation comptable et financière et la valeur créée pour l'actionnaire au niveau groupe; plutôt tournées vers la gestion des 
opérations, les systèmes d'information et la valeur créée pour le client au niveau des unités opérationnelles. La dimension financière du contrôle de gestion ne semble pas fondamentalement affectée par la différence entre industrie et service, le travail des contrôleurs de gestion au niveau groupe étant fondamentalement identique dans les services et l'industrie. Il n'en est manifestement pas de même pour la dimension opérationnelle du contrôle de gestion car le travail du contrôleur de gestion des unités de terrain a des particularités fortes dans le cas des activités de service. Rappelons à ce propos la définition générale que donne Bouquin (2006, p. 94) du contrôle de gestion : "Le contrôle de gestion est formé des processus et systèmes qui permettent aux dirigeants d'avoir l'assurance que les choix stratégiques et les actions courantes, seront, sont et ont été cohérents, notamment grâce au contrôle d'exécution. " (c'est nous qui soulignons). Nous nous situons donc dans une vision large du contrôle de gestion qui à nos yeux ne se réduit pas à la comptabilité de gestion et aux budgets mais va bien au delà.

Chenhall (2003, page 130) conseille de développer les recherches, qu'il estime encore trop limitées, sur le contrôle de gestion du secteur des services. Dans cette perspective, l'objet de cette contribution est de réfléchir aux méthodes et outils de contrôle de gestion liés à la montée en puissance des services dans les économies contemporaines. Des réflexions se sont déjà déployées au sujet du marketing des services (Lovelock et alii, 2008) et de la gestion des opérations (Heineke et Davis, 2007). Nous souhaitons faire de même pour le contrôle de gestion. Notre objectif, à cette étape, n'est pas d'aborder les diverses configurations de contrôle de gestion des entreprises de services comme des ensembles globaux, cohérents et différenciés contrairement à ce que préconisent Malmi et Brown (2008) et ce qu'avait tenté Bessire (1998) à partir de l'étude du commerce de détail et de la banque commerciale, mais de faire le travail préalable et nécessaire (qui a notre connaissance n'a jamais été fait sous cette forme) d'identification des particularités fondamentales du secteur, de recensement des enjeux spécifiques qui en découlent et des instrumentations liées et de mise en évidence des logiques de contrôle mises en œuvre. Pour cette raison nous privilégierons comme champ d'analyse les services à la personne plutôt que les services à l'entreprise et les services «purs » plutôt que les solutions combinant produits manufacturés et services liés que développent de plus en plus les industriels. Cette catégorie de prestations de services «purs » à la personne représente en effet la majeure partie des emplois du tertiaire marchand. Elle concentre la plupart des spécificités remarquables des services et constitue l'idéal-type de référence prédominant. Les préoccupations de contrôle de gestion qu'on y rencontre sont emblématiques de ce qu'on retrouve à un degré ou un autre dans la plupart des activités de service.

Dans une première partie de notre réflexion, nous étudierons la nature spécifique des activités de service et son impact sur les modes de gestion de la chaine de valeur servicielle. Dans une deuxième partie, nous mettrons en évidence les préoccupations du contrôle opérationnel des services qui en découlent et l'instrumentation associée. Dans une troisième partie, nous réfléchirons à la nature des systèmes globaux de pilotage de la performance dans les services et nous verrons comment ils intègrent les pratiques et outils précédemment identifiés. Ceci nous permettra de proposer une conception du contrôle de gestion dépassant la vision classique d'Anthony (1965) et faisant sa juste place à la dimension opérationnelle. Ainsi notre ambition est d'appréhender la réalité méconnue du contrôle de gestion des activités de service par un retour aux sources du contrôle de gestion. 


\section{La nature spécifique du management opérationnel des activités de service}

Les modalités du contrôle de gestion sont toujours étroitement liées à la nature de la fonction de production qu'on pilote. Pour appréhender et caractériser le contrôle de gestion des activités de service, il faut donc identifier les spécificités du secteur. C'est pourquoi, nous allons d'abord mettre en évidence les caractéristiques des services et leurs impacts sur la gestion des processus serviciels (1.1.) puis nous essaierons de dégager un cadre conceptuel de référence utilisable ensuite pour notre réflexion sur les modalités du contrôle (1.2.).

\subsection{Les caractéristiques distinctives des services}

Quatre caractères distinguent les services des activités industrielles : ils sont immatériels, hétérogènes, produits et consommés simultanément, non stockables (Van Looy, Gemmel et Van Dierdonck, 2003 ; Fitzsimmons et Fitzsimmons, 2008). Dans le secteur des services, le modèle d'affaires est composé de la cible commerciale (target market), des caractéristiques intrinsèques du service offert (service concept) et des modes de délivrance de la prestation (service delivery system choices) comme l'indiquent Roth et Menor (2003). Les modes de délivrance de la prestation de service étant au cœur de la réflexion en matière de contrôle de gestion des services, nous présenterons les traits fondamentaux des services en insistant à chaque fois sur leur impact sur la gestion des opérations et de la relation avec le client.

\section{1) Immatérialité des services}

Les services sont immatériels (intangibility). Ils ne consistent pas en la réalisation d'un objet physique mais en l'exécution d'un concept dans un acte ou un processus. Ceci a comme conséquence que, à l'inverse des biens physiques, les qualités intrinsèques des services ne peuvent que rarement être identifiées de façon sûre avant l'achat, ne se révèlent qu'en partie lors de l'expérience de consommation et pour la plupart restent difficilement évaluables même ex post par le client (Zeithaml, 1981). On peut penser par exemple au cas des diagnostics médicaux. Un élément essentiel de la gestion des services est donc de rendre tangible l'intangible par l'utilisation des supports physiques et des facteurs d'ambiance (Bitner, 2000). On peut aussi accroître la «ligne de visibilité » du client en dévoilant les processus productifs mis en œuvre pour le servir (par exemple dans un restaurant japonais en réalisant sous les yeux du client les phases de traitement du poisson cru, la préparation et la cuisson des ingrédients d'accompagnement, la confection des sushis, etc.) ou en rendant possible, par l'usage des technologies Internet, le suivi, à distance et de chez soi, de l'élaboration et de la réalisation progressive de la prestation (comme par exemple le suivi par le client de La Poste du parcours des lettres ou des colis).

La production de services nécessite l'implication du client dans ce qui est une co-production de la prestation qui associe l'offreur (entreprise) et le consommateur (client). Le degré de coopération peut être plus ou moins important mais dans tous les cas le personnel est amené à gérer simultanément deux faces de son métier : la gestion opérationnelle (l'utile pour le client) et la gestion relationnelle (l'agréable pour le client). En raison de l'immatérialité du service, la satisfaction finale du client dépendra en effet autant des conditions de réalisation de la prestation que du contenu même du service qui lui est délivré. 


\section{2) Hétérogénéité des services}

Les services sont beaucoup moins standardisés que les biens manufacturés produits en séries. La diversité des situations rencontrées par l'entreprise de service génère une très grande variété dans le processus de délivrance de la prestation de service (heterogeneity). Le service est un processus s'étendant dans le temps, il subit donc des variations en fonction du comportement du personnel de contact, des attentes et des réactions du client, des facteurs liés à l'environnement de la prestation. A côté de cette hétérogénéité subie, il y a aussi l'hétérogénéité choisie : la variété des prestations et l'adaptation aux demandes de la clientèle peuvent constituer un facteur clé de succès fondamental du modèle d'affaires de l'entreprise.

L'hétérogénéité intrinsèque de la prestation de service découle naturellement de ce qui vient d'être dit sur l'aspect relationnel de la prestation: en général, l'activité de service est plus orientée vers les gens que vers les choses. Le personnel de contact, maillon ultime de la chaine de valeur, est l'élément décisif de la prestation de service qui mobilise une proportion très importante de facteur humain (beaucoup plus que dans l'industrie où le capital technique est central). C'est pourquoi la formation, l'animation et le contrôle du personnel de contact sont des éléments majeurs de performance des activités de service.

\section{3) Coréalisation de l'offre de service avec le client}

Les services sont produits et consommés simultanément (inseparibility ou simultaneity). La production de service implique une interaction plus ou moins profonde de l'offreur et du client. La zone de contact peut être importante (high contact services) ou moindre (low contact services) mais la prestation est co-construite dans un processus dénommé « servuction » (Eiglier et Langeard, 1987 ; Eiglier, 2004). La Socialisation Organisationnelle du Client (SOC) est un élément fondamental pour réussir l'association du client dans le processus productif (Goudarzi et Eiglier, 2006). Comme le client doit être présent lors de la réalisation de la prestation, les points de délivrance du service se situent à proximité immédiate de la clientèle visée: les services sont la plupart du temps dépendants de l'emplacement et effectués dans le réseau des unités de terrain.

L'inséparabilité de la production et de la consommation de service a un impact certain en matière de gestion des opérations. La production des biens manufacturés se fait en usine dans un système fondamentalement fermé (la présence de stocks permettant de découpler la phase de production de la phase de commercialisation) même si ceci peut être nuancé quand on est dans le cadre d'une production en juste à temps où les flux productifs sont déclenchés par les commandes et donc «tirés par l'aval » (c'est pour cette raison que nous avons indiqué dans l'introduction que le Toyotisme fait entrer une partie des préoccupations de service dans l'univers manufacturier). Par opposition l'appareil de production des services est un système ouvert par nature. Si le gros problème de la gestion des opérations dans l'industrie est le problème des stocks de produits, la principale difficulté de la gestion des opérations dans les services réside dans la formation des files d'attentes et la gestion des «queues» de consommateurs attendant leur tour, ce que l'on peut analyser comme un «stockage des clients ». 


\section{4) Ajustement temporel de l'offre de service à la demande}

Les services sont non stockables (perishability). A l'inverse des biens manufacturés, les services ne peuvent pas être stockés à la fois en raison de leur nature immatérielle, de leur adaptation aux besoins spécifiques des différents clients et de la simultanéité de leur production et de leur consommation. Il faut donc s'adapter à une demande qui est souvent fluctuante et volatile. Cette adaptation ne concerne pas que la dernière maille de la chaine de valeur: en amont les actions de back office peuvent être d'un grand secours pour face aux aléas de la demande.

Le problème des pointes d'activité («coups de feu ») et des files d'attentes («queues ») est au cœur de la gestion opérationnelle des activités de service. Le non stockage est à la source de tous les problèmes inhérents aux services en raison d'une capacité de production limitée face à une demande variable et parfois imprévisible. De nombreuses réflexions en management des services portent sur la détermination de la capacité optimale (choix d'investissement) et sur les leviers d'action disponibles pour utiliser au mieux une capacité existante (gestion des opérations sur le terrain). Mais on peut aussi mobiliser des leviers d'action pour agir en amont sur la demande, la déplacer et l'écrémer (Meyssonnier, 2008).

Les quatre critères que nous venons de présenter sont assez proches du triptyque des caractères techniques par lequel certains auteurs francophones définissent les activités de service : immatérialité, interactivité, immédiateté (cf. à ce propos Djellal et Gallouj, 2007, page 10) mais s'y rajoute l'hétérogénéité qui est un élément essentiel quand on s'intéresse au contrôle de gestion des services. On peut construire des classifications des services à partir de tous ces critères : en fonction du degré d'immatérialité, du degré d'hétérogénéité ou du degré de simultanéité. On peut aussi introduire de nouveaux critères : l'importance relative de la phase de contact entre le personnel et le client; l'importance des fluctuations (anticipées ou non) de la demande; le degré de personnalisation des services; l'orientation des service plutôt vers les personnes ou plutôt relativement aux équipements ; etc. On peut enfin faire des typologies où on croise certains de ces facteurs deux à deux (Mills et Margulies, 1980 ; Maister, 1997). Cette diversité dans les approches des services amène tout à fait naturellement à se poser la question de l'existence (ou non) d'un cadre conceptuel global intégrant toutes ces dimensions et valable pour l'ensemble des activités de service.

\subsection{Un cadre de référence unifié difficile à trouver}

Le secteur des services est actuellement l'objet de mutations qui le transforment en profondeur. Ces évolutions majeures des activités de service interviennent dans trois dimensions : les modèles d'affaires se différencient et se juxtaposent (signe de maturité dans le cycle de vie des offres de service) ; les services se globalisent, deviennent multipartenaires et sont de plus en plus le fait de réseaux inter organisationnels ; enfin, grâce à la technologie (les automates et Internet), le client est de plus en plus impliqué dans la prestation, se passant même parfois du personnel de contact.

En effet, alors que l'offre de service a été pendant longtemps relativement homogène dans ses paramètres commerciaux et stratégiques avec des formes de prestations qui étaient généralisées successivement sur un marché, on assiste maintenant de plus en plus à la coexistence sur un même marché de chaines de valeur très différentes : par exemple une offre haut de gamme, une offre low cost et plusieurs offres intermédiaires dédiées à certaines cibles. On a donc plusieurs modèles d'affaires qui se concurrencent mais qui sont bien différents 
dans les caractéristiques de l'offre (services standards ou sur mesure par exemple) comme dans la clientèle visée (focalisation sur des segments distincts du marché global).

La nécessité pour les entreprises innovantes de service de croître rapidement face à la concurrence les amène aussi souvent, si elles doivent développer un réseau d'agences, de bureaux ou de points de délivrance de la prestation au voisinage de la clientèle, à choisir un mode de généralisation passant par la délégation ou l'association comme par exemple dans le cas des réseaux de franchise. Parfois, il faut faire aussi une offre globale au client englobant des prestations différentes mais liées sur la même chaîne de valeur (par exemple dans le tourisme). Dans toutes ces situations, se posent les problèmes de coordination et de contrôle de la qualité des prestations dans une offre combinée et solidaire qui est celle du système de service dans son ensemble.

Les technologies, et notamment les technologies de l'information, jouent enfin un grand rôle dans l'évolution récente du secteur des services. Le développement des automates fait que les clients sont de plus en plus actifs et impliqués dans la délivrance de la prestation mais avec une baisse de l'intensité de la phase d'interaction humaine avec le personnel de contact. C'est le cas quand le client passe par un standard téléphonique automatisé, quand il prend et paie tout seul son billet de train ou procède à des opérations au guichet automatisé d'une banque. C'est toujours le cas quand il se sert, pèse, enregistre et paie ses courses dans la grande distribution généraliste, en totale autonomie par rapport au personnel de caisse. L'irruption d'Internet bouleverse bien évidemment les repères dans le champ des services : le personnel de contact est absent, et c'est alors l'expérience du client lors de son passage sur un site et pendant l'usage du réseau virtuel qui est cruciale.

Ces modifications font que la définition des services par les quatre attributs évoqués précédemment devient de plus en plus difficile (car de nombreux services ne les respectent plus de façon exhaustive). Certains auteurs spécialistes du marketing des services indiquent aussi que la distinction entre production de services et production de biens, telle qu'elle est soulignée dans le modèle IHIP, est fragilisée car ces deux univers sont de plus en plus proches (Vargo et Lusch, 2004). La nécessité se fait jour de recentrer les fondements conceptuels définissant les services afin d'appréhender de façon globale et intellectuellement unifiée les services. Cette volonté est d'ailleurs au cœur du développement actuel d'effort interdisciplinaires visant à faire émerger une «Science des Services » qui permette de fédérer trois champs d'étude : les recherches en gestion (stratégies dans les services, marketing des services, gestion des processus productifs serviciels, pilotage de la performance dans les services, etc.) ; les recherches en technologie (conception des supports physiques mis en œuvre dans les processus en amont (back office) ou en aval (front office), ergonomie et design de l'accueil des clients, outils et systèmes informatiques, environnement Internet, méthodes de traitement de l'information, etc.); les recherches en sciences sociales (psychologie, sociologie, communication, comportements innovants, etc.). Des coopérations entre universités (Université of Cambrige (UK), University of California Berkeley (USA), North Carolina State University (USA), etc.) et grandes entreprises (IBM particulièrement) se développent autour de cette «Service Science, Management and Engineering (SSME)» en émergence.

Certains auteurs proposent un nouveau paradigme définissant les services comme de la création de valeur pour le client sans cession de droits de propriété (Lovelock et Gummesson, 2004). Pour eux les concepts de location ou de droits d'accès sont centraux dans la définition des services mais en raison de la grande diversité des situations doivent être précisés et la 
théorie discutée à partir d'études sur les sous-catégories de services afin d'éviter des généralisations abusives ou mal fondées. D'autres proposent d'élaborer une théorie unifiante des services autour de la place centrale du consommateur dans la co-construction de la prestation (Sampson et Froehle, 2006). Dans cette approche, ils reprennent la tradition qui fait de la relation de service (comme mode de coordination entre agents économiques) l'élément fondateur de ce type d'activités (de Bandt et Gadrey, 1994). Le service est défini comme l'ensemble des actes réalisés par le prestataire au profit d'un client, généralement avec sa participation en ayant pour objectif de transformer l'état d'un support. Il s'agit pour le prestataire de résoudre un problème qui concerne un support (bien matériel, information codifiée, connaissances ou l'individu lui-même). D'après Djellal et Gallouj (2007), cette prestation de service est la combinaison de différentes fonctions : matérielles (entretien ou transport d'un bien par exemple), informationnelles (production ou traitement d'une information codifiée comme le font un concepteur de logiciels ou un expert-comptable), relationnelles (service direct au contact du client comme dans une agence de voyage) ou méthodologiques (traitement des aspects cognitifs comme dans le cas d'un consultant en stratégie).

Ni l'une, ni l'autre de ces définitions n'est totalement satisfaisante et ne recouvre vraiment la globalité des activités de service. La proposition visant à faire du concept de «location » l'élément fondamental de définition des services polarise l'attention sur les caractéristiques intrinsèques du service offert alors que la proposition visant à faire du concept de «coproduction » l'élément central de l'identité des services focalise l'attention plutôt sur le mode de délivrance de la prestation. Gadrey (1997) écrivait il y a un certain temps, dans l'introduction d'un dossier de la Revue Française de Gestion consacré aux services : «Les critères de différenciation des situations de service et des modèles correspondants dépendent évidemment des questions que l'on souhaite traiter, des problématiques de recherche ou d'action. Il y a donc autant de façons de distinguer des modèles pertinents que de catégories de problèmes à résoudre. Une typologie n'a donc de sens qu'orientée vers des objectifs intellectuels : en matière de recherche aussi, la structure suit la stratégie, elle doit donc lui être adaptée. ». C'est pourquoi, à notre sens, c'est la définition des services à partir de la notion de co-production du service avec le client qui est la plus intéressante pour la réflexion en matière de contrôle de gestion serviciel car elle se concentre naturellement sur l'articulation coût-valeur dans les dispositifs de pilotage des processus productifs. Nous pouvons remarquer que c'est aussi au même fondement conceptuel qu'aboutissent Edvardsson et alii (2005) sur la base d'une revue de la littérature et de la consultation de onze experts parmi les plus connus du monde académique de la recherche sur les services.

Après cette réflexion sur la nature des activités de service, nous allons maintenant examiner les pratiques et outils de contrôle qui en découlent dans les services.

\section{Pratiques et outils du contrôle opérationnel des services}

Pour maîtriser les différentes dimensions spécifiques aux services, les entreprises du secteur se sont dotées d'outils d'analyse de la situation, de pilotage de la performance et de mise sous tension de l'organisation (Gervais, 2009). Sans méconnaître le développement des services liés aux biens et vendus conjointement que ce soit aux entreprises ou aux particuliers (approches en termes de «solutions » qui sont actuellement développées par bon nombre d'industriels), nous nous intéresserons plus particulièrement (pour les raisons déjà évoquées en introduction) aux activités de services «purs » aux particuliers (qu'ils concernent un 
support matériel extérieur, leur personne physique ou leur personnalité) parce qu'elles sont au cœur de l'économie des services (Bancel-Charensol et Jougleux, 1997). A l'intérieur même de l'organisation des entreprises de service, on peut identifier trois grands univers (Gadrey et Zarifian, 2001) : l'univers de la conception des nouveaux services (R \& D), l'univers des grands systèmes techniques et administratifs (plateaux techniques ou centres de traitement administratifs) et l'univers de la relation directe avec les clients (réseau commercial et platesformes téléphoniques). C'est dans la gestion du réseau et du personnel de front office que les spécificités du contrôle des activités de service sont les plus fortes.

Nous allons décrire les préoccupations emblématiques et les outils du pilotage de la performance des services qu'ils soient spécifiquement mis en œuvre par les contrôleurs de gestion (Ducrocq, Gervais et Herriau, 2001) ou activés par d'autres acteurs dans l'entreprise dans le cadre de leurs fonctions propres (voir à ce propos Lorino, 2008) et nous allons examiner comment ils s'articulent. Il nous semble en effet qu'on ne peut aborder le contrôle des activités de service sans une connaissance assez précise des préoccupations managériales spécifiques au secteur des services, de l'instrumentation de gestion qui en découle et aussi de la façon dont certains dispositifs, ne relevant pas apparemment du contrôle de gestion classique, sont particulièrement adaptés au contexte des prestations de service et contribuent à l'optimisation du pilotage de la performance dans ce secteur. Nous ne reviendrons pas sur les outils classiques du contrôle de gestion (calculs de coûts et budgets) utilisés par les contrôleurs de gestion dans toutes les entreprises (quoique qu'on doive remarquer que les calculs de coûts dans les services ont longtemps été beaucoup moins développés que dans l'industrie). Nous nous intéresserons aux instruments dédiés aux activités de service, souvent à la frontière du contrôle de gestion et d'autres fonctions et utilisés aussi bien par les managers de terrain que par les contrôleurs de gestion.

Nous allons dans un premier temps recenser les enjeux les plus significatifs à nos yeux en matière de contrôle des activités de service et évoquer l'instrumentation liée (2.1.) puis nous examinerons le degré d'usage et les facteurs de contingence dans la mise en œuvre de ces pratiques et outils du contrôle (2.2.).

\subsection{Objectifs et instrumentation liée}

Il nous semble possible de distinguer les préoccupations particulièrement importantes dans les activités de service et de présenter l'instrumentation qui va avec. Nous avons mis en évidence six démarches emblématiques du contrôle opérationnel des services (avec des liens entre elles sur lesquels nous reviendrons ensuite).

\section{1) Identifier les unités les plus efficientes d'un réseau}

La plupart des entreprises de services sont organisées en réseaux d'unités comparables délivrant les prestations de service au plus près des clients. Mais ces unités de base ne sont jamais totalement similaires car la composition du bouquet de prestations (outputs) et la structure des consommations de ressources (inputs) varie notamment en fonction de la clientèle. On peut mesurer la productivité de ces différentes unités par la mise en œuvre de la méthode DEA (Data Envelopment Analysis). Cette méthode de programmation linéaire, particulièrement bien adaptée aux entreprises de service gérant des agences, magasins ou bureaux comparables sur un vaste territoire, permet de déterminer les unités les plus efficientes dans un réseau multi-sites et multi-activités et de quantifier les écarts entre les unités d'excellence et les moins performantes (Metters, Frei et Vargas, 1999). On connaît les 
unités performantes et les unités en situation plus critiques même si ceci ne nous dit pas les raisons du différentiel constaté d'efficience (de La Villarmois, 1999). On a là une voie intéressante (quoique pas encore pleinement exploitée) pour mesurer la productivité des activités de service (Gervais et Thenet, 2004). On peut même combiner des mesures de la performance «opérationnelle» fondée sur la bonne utilisation des ressources dans la production et de la performance «financière » attachée aux coûts et aux marges des activités (Deville et Leleu, 2010).

Cet outil d'analyse sert en contrôle de gestion comme une première étape dans la mise en place d'une démarche de benchmarking interne approfondi pour trouver les raisons des différences constatées de performance. Le travail du contrôleur de gestion sera ensuite d'identifier les meilleures pratiques et de donner les moyens aux unités les moins performantes de s'aligner sur le comportement des unités les plus performantes. Dans les activités commerciales et de service ceci représente une part très importante du travail du contrôleur de gestion.

\section{2) Généraliser les meilleurs processus de servuction}

Après avoir identifié les unités de référence (par la méthode DEA), le contrôleur de gestion des entreprises de service va étudier les pratiques managériales, les chaînes de valeur et l'articulation des tâches dans les processus serviciels afin de comprendre les ressorts de la performance des entités locales au contact du client. Il s'agira ensuite de formaliser ces modes opératoires et de standardiser les procédures afin de généraliser la one best way ainsi mise en évidence. Un certain nombre d'acquis des activités industrielles en matière de méthodes de production ont été transférés aux activités de service afin d'assurer la traçabilité et d'accroître l'homogénéité des prestations délivrées. La production et la consommation étant simultanées dans les services, il ne peut y avoir de contrôle a posteriori : il doit être effectué avant et pendant la prestation. En 1978, Sasser élaborait des logigrammes intitulés flow charts qui décrivaient les différentes étapes des processus et les zones d'interfaces avec le client (Sasser et alii, 1978). Ceci a été approfondi par tout un courant de recherche et a abouti au blueprint de Schostack (1992). Ce document fournit une description complète du mode de délivrance de la prestation avec identification des différentes phases de la prestation de service et des zones à problèmes. Les actions se déroulent dans le temps de haut en bas de la représentation graphique et on voit clairement de gauche à droite ce qui relève respectivement: du back office; des supports physiques, artefacts et outils; des actions du personnel en contact ; des actions du client. A partir de là, on peut identifier les points d'échec potentiels et essayer d'anticiper ou d'éviter ces problèmes qualifiés d'OTSU (Opportunity To Screw Up) par Maister (2001) ou encore de modifier l'organisation des processus dans une démarche de redesign (Berry et Lampo, 2000). Il est même possible d'aller plus loin et de tenter de représenter le schéma mental des attentes du client nourri par ses expériences précédentes. Ce document, appelé script, est en quelque sorte le blueprint des servuctions attendues par le client (Orsingher, 2006).

Après avoir normalisé la prestation de service ex ante, il faut gérer, en situation, la diversité de la demande aussi bien qualitativement (composition hétérogène et variable de la demande) que quantitativement (pointes d'activités anticipables ou subies). 


\section{3) Gérer et contrôler les temps et les délais}

Dans le contrôle opérationnel des prestations de service, on doit faire face comme dans les activités industrielles au problème de la gestion conjointe du triplet coût-qualité-délais, mais c'est la dimension temporelle qui est probablement l'élément crucial dans les services. Le temps est à la fois un input (ressource consommée) et un output (prestation délivrée) dans les activités de service. Le temps est une source de coût (rémunération de la main d'œuvre) mais aussi parfois une source de revenus (quand le service est facturé à la durée). Le temps est un élément de non qualité, facteur de destruction de valeur pour le client (quand il s'agit d'un temps d'attente ou d'une durée trop longue de délivrance de la prestation) mais aussi de qualité perçue, contribuant à créer de la valeur pour le client (quand le personnel de contact s'adapte aux demandes de la clientèle et gère la relation autant que les opérations au rythme et en fonction des attentes de la clientèle). La prise en compte subjective des délais par les consommateurs est un élément souvent décisif dans la qualité perçue. Bitran, Ferrer et Rocha e Oliveira (2008) insistent pour que la dimension temporelle soit au centre des préoccupations des gestionnaires des opérations.

On peut quantifier et contrôler le temps de réponse et d'action de l'entreprise (delivery time), par différents systèmes de mesure (Klassen, Russel et Chrisman, 1998). Depuis quelques années des innovations de gestion vont dans ce sens et semblent particulièrement adaptées aux services comme la méthode TD-ABC (Time Driven - Activity Based Costing) par exemple. La méthode de calcul des coûts fondée sur la prise en compte des temps TD-ABC, développée récemment par Kaplan et Anderson (2008), s'appuie sur la détermination des standards en matière de temps alloués aux opérations et aux processus (ceci peut venir dans la continuité des pratiques de benchmarking présentées au point précédent), grâce à l'établissement d'équations de temps correspondant aux différentes prestations et permet la mise en œuvre d'un suivi chiffré des réalisations au travers de budgets qui sont l'expression monétaire de cette consommation de la ressource temps. Certaines études récentes mettent toutefois en évidence les limites de l'outil (Allain, 2010).

De nombreuses techniques sont mobilisées par ailleurs pour gérer les files d'attente et les délais perçus par les clients (waiting time) comme le rappelle une synthèse des travaux de recherche sur cette question effectuée par Durrande-Moreau (1999). On développe en effet dans les services toute une panoplie de méthodes pour diminuer les temps d'attente (Meyssonnier, 2008) par la réorganisation du dispositif d'accueil et de traitement des clients (ce qui peut passer selon les cas par le développement de la polyvalence ou au contraire de la spécialisation), en impliquant le client dans la préparation de la délivrance de la prestation avant même la phase de contact au guichet (distribution de dossiers, formalisation anticipée de la demande, orientation différenciée des clients en fonction de la nature de la demande, etc.) ou en faisant que l'attente par le client ne soit plus du temps «mort » (par la distribution de tickets, le développement d'activités parallèles pendant le temps d'attente, etc.).

Par delà ces ajustements, la question de l'adéquation structurelle entre demande et capacité est centrale dans les activités de service où le stockage est impossible.

\section{4) Gérer la demande et adapter les capacités}

C'est là un enjeu majeur des activités de service (Crandall et Markland, 1996). Pour utiliser au mieux les capacités et optimiser les recettes, une démarche de yield management est souvent mise en œuvre dans les entreprises qui présentent les caractéristiques suivantes : un 
stock périssable ou une impossibilité de stockage, une demande variable face à une capacité fixe, des ventes par réservation, des coûts marginaux faibles (Kimes et Chase, 1998). Elle permet de déplacer la demande par le transfert de la clientèle des périodes saturées vers les périodes où l'offre est disponible et de l'écrémer par l'augmentation des tarifs en périodes de pointe (Weatherford et Bodily, 1992 ; Capiez, 2003). On peut aussi combiner cela avec des pratiques de surbooking.

Cette gestion des revenus dépasse le cadre des fonctions traditionnelles. Basée sur l'usage intensif des bases de données et des séries statistiques, elle associe les spécialistes du marketing, ceux de la gestion des opérations et les responsables financiers et du contrôle de gestion. Plus les coûts fixes seront élevés et les coûts variables faibles, plus la démarche de yield management sera pertinente et ses apports à l'amélioration de la marge de l'entreprise significatifs.

Si les actions marketing ne suffisent pas, on doit agir sur la flexibilité de la capacité de production (Klassen et Rohleder, 2001). On a en effet tout un champ d'actions possibles pour gérer la structure de production (capacity management) dans les services. Après avoir déterminé les capacités nécessaires dans une approche en terme de seuil de rentabilité, on optimisera le fonctionnement des capacités installées.

\section{5) Mesurer la qualité de service}

La satisfaction du client et la valeur perçue, ne dépendent pas que de la qualité objective du service délivré (mesurable par des indicateurs internes précis et «durs ») mais aussi de la perception subjective qu'a le client de l'adéquation de la prestation vécue avec son désir (mesurable uniquement par des indicateurs externes plus généraux et «mous»). Cette dernière dimension est au cœur des modèles de mesure de la satisfaction dans les services élaborés à partir de 1985 par Parasuraman, Zeithaml et Berry. L'évaluation de la perception de la qualité du service par le client se fait autour de 5 dimensions : l'effectivité supposée (apparence du contexte matériel et des éléments physiques de support entourant la prestation), la fiabilité (respect des engagements, performances sérieuses et régulières), la réactivité (aide et promptitude face aux aléas), l'assurance (compétence, courtoisie, crédibilité, sécurité) et l'empathie (accès facile, bonne communication, compréhension du client). L'outil de mesure Servqual permet d'évaluer la satisfaction du client. Une identification des écarts entre les attentes du client et sa perception de la prestation (gaps model) est également réalisable. A ce propos on peut mettre en évidence une «zone de tolérance » du client à l'intérieur de laquelle il ne prête pas d'attention à la variation dans le niveau de performance de certains attributs du service (Johnston, 1995). Dans la période récente le modèle Servqual est toutefois parfois critiqué (Morrison-Coulthard, 2004).

La mesure de la qualité peut sembler un outil relevant plus du marketing que du contrôle de gestion. Mais le lien entre la valeur perçue par le client et les coûts engagés par l'entreprise est au cœur de nombreux débats en contrôle de gestion. La volonté de construire un pont entre les coûts et la valeur est consubstantielle à un certain nombre d'outils comme le target costing (Meyssonnier, 2001) ou l'ABC (Mac Nair et alii, 2001). Par exemple, Cugini, Caru et Zerbini (2007) présentent et discutent un cas de mise en relation des coûts et de la valeur perçue dans une activité de service. Certains auteurs doutent pourtant de la possibilité de réussir cette connexion (Malleret, 2009). 


\section{6) Mettre sous tension l'entreprise de service}

Dans les activités de service, on gère une relation autant que des opérations et on co-construit la prestation avec le client. Ceci amène à devoir contrôler un spectre plus large d'éléments que dans l'industrie classique afin d'assurer la convergence des comportements des membres de l'entreprise. Il semble donc qu'un outil comme le balanced scorecard (Kaplan, 2009), tableau de bord stratégique multidimensionnel regroupant divers indicateurs relatifs à toutes les dimensions d'action de l'entreprise (aspects financiers; aspects commerciaux et de satisfaction du client; aspects relatifs aux processus de transformation et à la chaine de valeur; aspects liés aux connaissances et aux savoir-faire) est particulièrement adapté au contexte des activités de service. Au cour du balanced scorecard, on a des chaînes de causalité qui partent des paramètres opérationnels et cheminent jusqu'aux facteurs clés de succès identifiés dans une carte cognitive partagée par les managers. Ces liens de causes à effets génèrent de la performance financière si la vision stratégique subjective est validée objectivement par le marché. La diversité des paramètres à gérer conjointement au niveau du personnel de contact peut être appréhendée grâce à cet outil. Dans le secteur des services, les tableaux de bord sont au cœur du pilotage de la performance (probablement plus que les budgets) et le lien entre les indicateurs opérationnels (mesure avancée de la performance en train de se construire) et les indicateurs financiers (mesure retardée de la performance constatée) se fait particulièrement bien dans le cadre du balanced scorecard. On articule ainsi le contrôle stratégique au contrôle opérationnel sans passer par l'outil central habituel du contrôle de gestion : la modélisation financière des budgets est reléguée au second plan.

\subsection{Contextes et usages de l'instrumentation}

Nous pouvons synthétiser les principales caractéristiques et techniques du contrôle de gestion des activités de service, que nous venons d'évoquer, dans le tableau ci-après.

Tableau relatif à l'instrumentation du contrôle des prestations de services

\begin{tabular}{|c|c|c|}
\hline & $\begin{array}{l}\text { Principaux instruments } \\
\text { de contrôle opérationnel } \\
\text { des services }\end{array}$ & $\begin{array}{c}\text { Facteurs de contingence } \\
\text { favorables à la généralisation } \\
\text { de la pratique managériale ou de l'outil }\end{array}$ \\
\hline 1) & $\begin{array}{l}\text { détermination des unités les plus efficientes } \\
\text { par la méthode } D E A\end{array}$ & $\begin{array}{l}\text { - organisation en réseau d'unités identiques } \\
\text { - forte densité de l'implantation des unités sur le terrain }\end{array}$ \\
\hline 2) & $\begin{array}{c}\text { benchmarking } \\
\text { et contrôle du processus par le blueprint }\end{array}$ & $\begin{array}{l}\text { - importance de la phase de contact } \\
\text { - service homogène pour une clientèle de masse }\end{array}$ \\
\hline 3) & $\begin{array}{l}\text { gestion des temps productifs, } \\
\text { évaluation des coûts par le } T D-A B C, \\
\text { puis gestion des files d'attente }\end{array}$ & $\begin{array}{l}\text { - co-production avec le client } \\
\text { - rapidité du service fortement valorisée par le client }\end{array}$ \\
\hline 4) & $\begin{array}{l}\text { action sur la demande par le yield management, } \\
\text { puis gestion des capacités }\end{array}$ & $\begin{array}{l}\text { - pas de stockage } \\
\text { - coûts fixes élevés et coûts variables faibles }\end{array}$ \\
\hline
\end{tabular}




\begin{tabular}{|c|c|c|}
\hline 5) & $\begin{array}{c}\text { mesure de la qualité subjective par servqual, } \\
\text { puis mise en relation avec les coûts }\end{array}$ & $\begin{array}{c}\text { - gestion des opérations mais aussi de la relation } \\
\text { - complexité et diversité de la prestation }\end{array}$ \\
\hline 6) & mise sous tension par le balanced scorecard & - importance du personnel de contact \\
\end{tabular}

Ces méthodes et outils dont les caractéristiques techniques sont bien adaptées aux particularités des services ont des degrés de diffusion variables. La méthode DEA a été l'objet de nombreux travaux académiques dans le secteur bancaire et dans la distribution (mais manifestement de moins d'applications professionnelles). L'usage des blueprints est classique dans tous les services à la personne (restauration commerciale, garages, etc.) et est parfois conforté par des codes déontologiques ou des règles professionnelles (dans la santé, les services financiers, etc.). La gestion des files d'attente est maintenant généralisée dans les services mais l'outil TD-ABC qui permet de calculer des coûts sur la base des temps d'activité mesurés est récent et encore très peu répandu. Le yield management et la gestion des capacités sont tout à fait courants dans les transports (depuis trente ans dans le transport aérien, depuis une dizaine d'années dans le transport ferroviaire) et se sont progressivement généralisés dans l'hôtellerie, la location de voitures et les activités de service analogues. Les questionnaires et enquêtes de satisfaction sont répandus partout dans les services mais le lien avec les coûts engagés mesurés par l'ABC est encore assez rare. Les balanced scorecards sont maintenant habituels dans la plupart des entreprises de service depuis une dizaine d'années.

Si on devait distinguer, comme le préconisent Malmi et Brown (2008), entre les outils d'aide à la décision et ceux qui relèvent plus de l'orientation des comportements, on devrait mettre la méthode DEA, les gestions des revenus et des capacités et la mesure servqual de la qualité perçue dans la première catégorie (aide à la décision) et le blueprint, la gestion des temps et des délais et le balanced scorecard dans la deuxième catégorie (orientation des comportements). Mais en même temps on voit bien le lien entre ces éléments par paires : la méthode DEA permet le benchmarking des processus et l'élaboration des logigrammes ; la gestion des temps et des délais met en évidence les contraintes insurmontables et appelle le yield management et la gestion des capacités ; l'évaluation de la qualité perçue par le client (servqual) ouvre la voie au pilotage des leviers d'action contribuant à cette valeur pour le client (balanced scorecard). Il semble donc vain de vouloir opposer ces deux variétés d'outils tant elles sont interdépendantes.

Ces pratiques et outils dédiés aux services ayant été identifiés, il s'agit maintenant d'étudier leurs logiques de mise en œuvre et leur intégration à des dispositifs globaux cohérents de pilotage.

\section{Les systèmes de pilotage de la performance des activités de service}

Nous allons nous appuyer sur des recherches récentes en matière de contrôle des activités de service afin de voir la façon dont les diverses préoccupations et instrumentations liées que nous venons de mettre en évidence s'articulent logiquement (3.1.) puis nous proposerons une 
réflexion synthétique sur la nature du contrôle de gestion s'appuyant sur l'exemple des services mais valable bien au delà (3.2.).

\subsection{Apports de recherches récentes sur les modes de contrôle dans les services}

Depuis les travaux pionniers de Hayes et Wheelwright (1979) sur les matrices de processus productifs, de nombreux auteurs ont développé des approches typologiques, fondées sur la mise en évidence de configurations de référence, dans les services. A ce jour le travail le plus abouti semble être la proposition de positionnement matriciel des services de Collier et Meyer (1998) mettant en relation deux concepts : la nature de la demande du client et les modes de délivrance de la prestation de l'entreprise de service. Le consommateur souhaite soit une prestation unique et ajustée à des besoins spécifiques élevés, soit des prestations nombreuses, avec peu de spécificités et un niveau d'exigence réduit. Le système de délivrance de la prestation peut être peu normalisé car s'adressant à une clientèle diversifiée ou bien fortement contrôlé car visant un segment particulier. Ceci conduit à mettre en exergue deux positionnements extrêmes : l'un orienté vers la demande (adapté aux besoins divers d'une clientèle exigeante), l'autre s'appuyant sur l'offre (capable d'assurer une prestation homogène et calibrée auprès d'une clientèle ciblée et peu exigeante).

Une étude du contrôle dans la restauration commerciale (Goureaux et Meyssonnier, 2011) a permis de mettre en évidence les différences entre établissements dans la gestion conjointe des coûts, de la qualité et des délais en fonction du modèle d'affaires adopté. Elle aboutit à des résultats qui confortent et actualisent les constations de Collier et Meyer. Une représentation graphique en est proposée ci-dessous (schéma 1).

\section{Schéma 1}

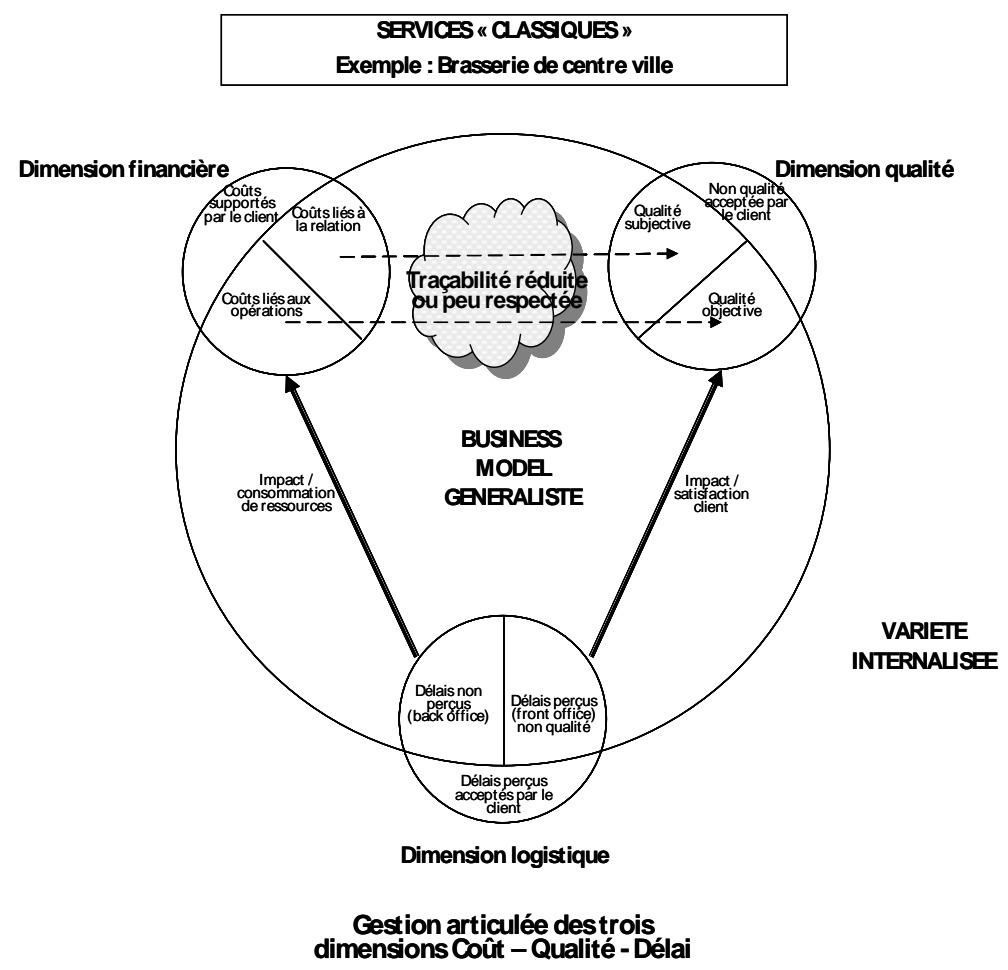

(Goureaux et Meyssonnier, 2011) 
Dans les services généralistes classiques, la variété est internalisée et gérée par l'entreprise. La traçabilité des processus est relativement réduite ou pas totalement respectée. Les ajustements sont réalisés in situ par les managers ou le personnel de contact. Pour ces raisons les leviers de performance sont essentiellement la formation et la capacité d'adaptation du personnel de contact dans la dernière maille de la chaîne de valeur de l'entreprise.

Ces éléments pourront être suivis dans le cadre du balanced scorecard en partant des indicateurs de l'axe « relation avec le client » (satisfaction subjective mesurée par servqual) et des indicateurs de l'axe «processus productifs» (mesure des coûts opérationnels, de la qualité objective et des délais) pour remonter jusqu'à l'axe «apprentissage organisationnel » avec les indicateurs avancés relatifs au personnel productif (de contact, bien sûr, mais aussi de support) : mesures de la capacité de polyvalence des équipiers, indicateurs de mise à jour technique des salariés, indicateurs d'accroissement des capacités relationnelles du personnel de contact, recensement des formations aux nouvelles technologies, à la gestion du stress ou des crises, etc. On sera alors plus dans une démarche de création de potentiel que de contrôle des tâches.

Par contre, il est possible de trouver des cas où le modèle d'affaires n'est plus généraliste mais centré. La variété prise en charge par l'entreprise est alors limitée, comme dans le cas des compagnies low cost, par réduction des engagements de l'entreprise et par ciblage de la clientèle. Le périmètre de ce qui relève vraiment de l'entreprise est moindre que dans le cas précédent (ce qui est clair dans le schéma 2 ci-dessous).

\section{Schéma 2}

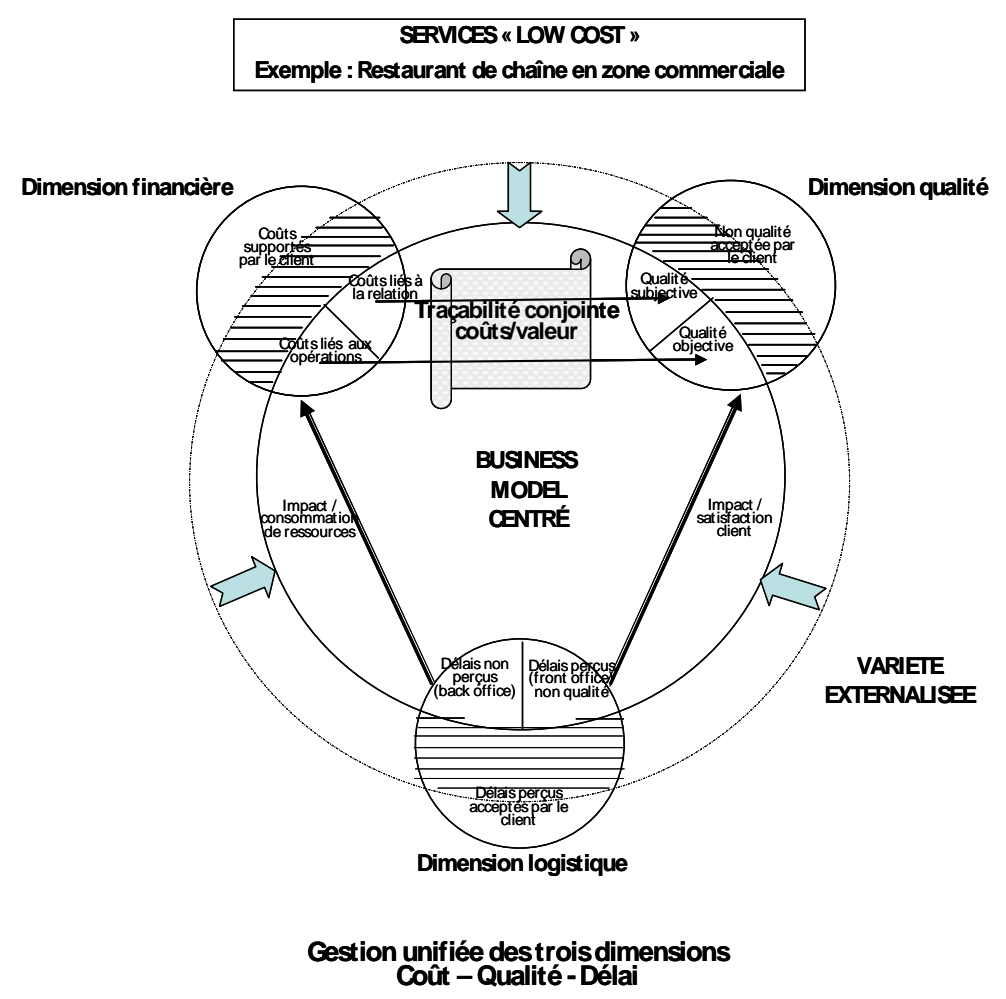

(Goureaux et Meyssonnier, 2011) 
Dans ce cas, la variété est externalisée : sur la chaîne de valeur de la prestation de services, la frontière entre ce qui est pris en charge par l'entreprise et ce qui est assumé par le client se déplace réduisant la part du travail imputable à l'entreprise. La traçabilité conjointe coûtvaleur semble alors possible dans le cadre de l'activité de l'entreprise. Dans cette situation, les leviers de performance sont essentiellement liés à détection des unités les plus efficientes, à la formalisation des processus par le blueprint et à la mise en œuvre de l'instrumentation de gestion interne consacrée à la gestion des temps, des délais, de la demande et des capacités (éléments que nous avons amplement vus dans la deuxième partie de cet article). Et on retrouve ici la vision de Chiapello (1996) pour qui le contrôle peut se comprendre comme «processus créateur d'ordre».

Cette approche consacrée au contrôle opérationnel de la chaîne de valeur converge avec la vision de Muriel Jougleux (2006) en marketing des services. Elle propose de distinguer dans la qualité d'un service, au sens large de satisfaction globale (même si nous savons qu'il faut distinguer la qualité perçue liée au service et la satisfaction proprement dite qui dépend des affects du client comme le souligne Olivier, 1996), ce qui relève de la qualité de service et ce qui relève de la qualité du service. Pour elle, plus la personnalisation du service est importante et plus les unités locales sont directement responsables de la satisfaction du client. La qualité

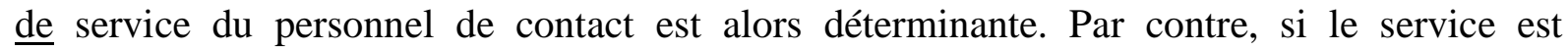
standardisé aussi bien dans son processus de production que dans son output, les marges de manœuvre du personnel de contact dans la dernière maille de la chaîne de valeur sont limitées. La qualité du service est totalement spécifiée et c'est par le respect des normes fonctionnelles et le contrôle opérationnel que la satisfaction du client est garantie. Le contrôle de gestion joue alors un rôle central dans la recherche de la performance.

Comme nous l'avons évoqué dans la première partie, deux autres évolutions impactent les contrôle de gestion des services : le développement des partenariats sur la chaîne de valeur globale et le rôle croissant des nouvelles technologies.

De plus en plus, l'offre de service au client est globale et donc les entreprises de service proposent un bouquet de prestations liées, articulées les unes aux autres dans un ensemble cohérent et complémentaire (cf. par exemple les circuits organisés dans le tourisme avec un voyage en avion, des lieux de résidence et des occupations régulières, la proposition d'excursions, d'activités spéciales optionnelles, la réalisation d'évènements, etc.). Le contrôle ne sera plus intra organisationnel, stable et formalisé mais inter organisationnel, adaptatif et plus ou moins formalisé. La mise en œuvre du contrôle de gestion dans les ensembles coopératifs structurés autour d'une chaine de valeur partagée est une des nouveautés actuelles du pilotage de la performance dans les services. Ainsi, une étude effectuée récemment par Goullet et Meyssonnier (2011) a montré que dans le cas des réseaux de franchise de services, le contrôle inter organisationnel $\mathrm{du}$ franchiseur sur le franchisé utilisait trois leviers principaux : un contrôle de conformité dans la réalisation des opérations, un contrôle de gestion plus centré sur l'efficacité que sur l'efficience et un contrôle social. Par ailleurs au fil du cycle de vie de la relation le poids respectif de chacun de ces modes de contrôle évoluait.

Dans l'aspect contrôle de conformité, on voit bien comment le benchmarking dans le réseau (notamment par alignement des franchisés sur les succursales de référence possédées par le franchiseur), le recours aux blueprints, le contrôle des délais et de la qualité de service par des inspecteurs techniques et des clients «mystère » envoyés par le franchiseur, le traitement centralisé des réclamations des clients, etc. trouvent leur place. Ainsi ces divers éléments 
(évoqués dans la partie 2 de cet article) s'intègrent bien dans un système global et cohérent de contrôle.

La technologie change aussi beaucoup et devient un des traits fondamentaux du secteur des services. Le personnel de contact est de plus en plus concerné par le développement des technologies de l'information et de la communication. Une recherche menée dans les services publics de proximité et focalisée sur la gestion des temps et des délais (Meyssonnier et Tahar, 2011) a mis en évidence, entre autres, ce rôle croissant des nouvelles technologies de l'information et de la communication dans les relations avec les usagers. Une visibilité pénétrante est aussi possible en temps réel sur le comportement du personnel en contact avec la clientèle. Les tâches physiques et les activités mentales sont de plus en plus lourdes ce qui amène dans de nombreux cas à développer un middle office entre le back office et le front office. Ce middle office joue un rôle de médiateur, soutien et superviseur (Baranger et alii, 2009). Les managers de terrain peuvent ainsi venir en renfort du personnel de contact des services taylorisés en cas de nécessité ou fournir un appui de type «boîte à outils » dans les services de type projet. Mais le développement de l'implication du consommateur dans la coréalisation de la prestation en s'appuyant sur les nouvelles technologies peut également amener à diminuer l'importance du personnel de contact. Le développement des services en ligne permet des traitements à distance (hot line) voire la disparition de certains des acteurs humains dans la maille de contact (standards téléphoniques informatisés pour les demandes de renseignement ou la réalisation de tâches à distance; passage par des caisses totalement automatisées dans les grandes surfaces de distribution; automates dans les banques ou dans les transports publics). Pour assurer la qualité de fonctionnement des automates, c'est le personnel de back office qui a alors un rôle décisif à jouer dans la programmation, la sécurisation et la continuité technique du service. Le recours de plus en plus fréquent aux technologies de self service diminue l'importance des aspects managériaux du pilotage de la performance, les hommes étant remplacés par des machines ou des systèmes informatiques actionnés directement par les clients. La régulation automatisée des nouvelles technologies se substitue de façon croissante aux dispositifs humains visant à assurer la convergence des comportements, rejoignant ainsi des constatations anciennes de Joan Woodward sur l'impact de la technologie sur les modes de régulation managériaux.

La mise en évidence de ces logiques d'action et de leur outillage amène naturellement à se poser la question de la nature même du contrôle de gestion des services.

\subsection{Repenser le contrôle de gestion des activités de service}

Au fil de notre analyse nous avons développé une approche large du contrôle de gestion des activités de service dépassant la seule modélisation financière en œuvre dans les différentes techniques de calcul et d'analyse des coûts (coûts complets ou coûts partiels) ou dans le système budgétaire (prévisions, reporting mensuel, analyse d'écarts et actions correctives).

Il nous semble qu'il est nécessaire de remettre en cause la vision classique d'Anthony (1965) qui distinguait la planification stratégique, du contrôle de gestion et du contrôle des tâches. Comme le remarque Otley (p. 364, 1999) à propos de la classification d'Anthony: «Le contrôle opérationnel a été négligé car il était clair que les différentes organisations utilisaient des pratiques différentes au niveau opérationnel et Anthony s'est concentré sur les éléments communs à toutes ces organisations. Se focaliser sur ces éléments communs permettait de développer un langage universel capable d'englober tous les types d'activités organisationnelles. ». Et il rajoute plus loin : «Bien qu'il ait été peut-être correct de se 
concentrer initialement sur le cour du champ "contrôle de gestion », il est maintenant nécessaire d'accorder plus d'importance aux aspects négligés de la stratégie et de l'opérationnel. ». Ceci l'amène aussi à insister sur la nécessité de passer d'une réflexion centrée sur la mesure de la performance (ex post) à une réflexion consacrée au pilotage de la performance (ex ante). Nous partageons tout à fait cette vision des choses. Et en prenant le champ des activités de service à la personne on a, nous semble-t-il, le bon niveau d'analyse pour appréhender, de façon contingente mais avec un assez grand degré de généralité, la dimension opérationnelle du contrôle de gestion. Les fondements du cadre de réflexion utilisés pour une telle démarche sont bien évidemment à trouver plus dans les approches du management fondées sur une vision à long terme des ressources et la valeur (Activity-Based Management ou Value-Based Management) que dans celles fondées sur la théorie de la firme, les relations d'agence et la maximisation à court terme des flux financiers de type Economic Value Added (EVA).

Plusieurs travaux ont été consacrés aux Systèmes de Pilotage de la Performance (SPP en français, PMS en anglais pour Performance Management Systems). Les systèmes de pilotage de la performance (SPP dorénavant) sont globaux et comprennent: la formulation des objectifs stratégiques et des indicateurs finaux de réussite ; la mise en évidence des chaines de causalités qui permettent de les atteindre et des critères de mesure du déploiement de la stratégie au niveau des activités ; la détermination des niveaux de performance requis dans chaque centre de responsabilité pour atteindre les objectifs de l'entreprise ; le système incitatif d'intéressement des managers et des employés couplé à la déclinaison des objectifs dans l'organisation; la détermination des flux d'information nécessaire pour mettre en œuvre la stratégie et pour l'adapter aux modifications de l'environnement. Otley (1999) a défini d'abord les SPP autour de 5 questions fondamentales, puis Ferreira et Otley (2009) ont proposé une analyse plus fine autour de 12 questions ce qui leur permettait d'intégrer les apports de Simons (1995) sur les leviers du contrôle. Cette approche des SPP a été critiquée par Broadbent et Laughlin (2009) qui estiment qu'il s'agit d'une tentative de présentation d'une voie moyenne susceptible de s'appliquer à l'ensemble des SPP mais qu'en réalité on a affaire à deux types de SPP : l'un qu'ils qualifient de «transactionnel » qui s'appuie sur une rationalité instrumentale, l'autre qu'ils qualifient de «relationnel» qui s'appuie sur une rationalité de communication. Il est clair que notre approche conceptuelle se situe fondamentalement dans le premier courant correspondant à une vision rationaliste qui est fondée sur l'analyse des outils particulièrement adaptés ou vraiment spécifiques des services : la méthode DEA et les blueprints pour déterminer et mettre en œuvre les bonnes pratiques ; la batterie des outils liés à la gestion des temps et des délais d'une part, au yield management et à la gestion des capacités d'autre part qui sont essentiels dans les services ; la mesure de la qualité de service servqual et le balanced scorecard qui permettent d'identifier ce qui est important pour le client et de mettre ensuite sous tension l'organisation pour le satisfaire.

Bien entendu, dans les services comme ailleurs, la mobilisation et l'appropriation des outils ne relève pas que d'une logique de rationalité pure, les aspects socio-politiques et psychocognitifs sont aussi importants. Mais il nous semble essentiel, si on veut dépasser une vision étriquée du contrôle de gestion, d'intégrer à la réflexion toute l'instrumentation servicielle qui façonne largement les comportements et les représentations. Et c'est à partir d'une approche technique et de prise en compte de certains facteurs de contingence que nous pouvons ensuite constater et/ou préconiser l'usage de leviers de contrôle adaptés. Ainsi par exemple, dans la restauration commerciale, on peut standardiser les comportements par les blueprints ou à l'inverse développer l'autonomie du personnel de contact. Dans les franchises de service, on peut utiliser de façon modulée dans le temps le contrôle de conformité des tâches, le contrôle 
de l'efficacité de l'exploitation et le contrôle social pour encadrer les activités du franchisé. Dans les services publics de proximité, on doit minimiser les délais quand ils sont uniquement un coût pour la mairie et une contrainte pour l'usager mais on peut se contenter d'optimiser les temps quand ils sont certes un coût pour la mairie mais par ailleurs une valeur pour l'usager.

\section{Conclusion}

Nous avons souligné l'importance des services dans l'économie contemporaine et présenté les caractéristiques des prestations de service. Les quatre principaux attributs des services (aspect immatériel ; hétérogénéité des situations dans la délivrance des prestations ; co-réalisation de la prestation avec le client; adaptation permanente aux fluctuations de la demande et gestion des files d'attente) expliquent certains traits du management opérationnel dans le secteur. Nous avons indiqué que le cadre conceptuel de référence fondé sur la co-production du service avec le client était probablement le plus pertinent pour appréhender le contrôle de gestion des activités de service.

Nous avons décrit six préoccupations emblématiques des activités de service et outils de contrôle de gestion liés. Ces dispositifs permettent respectivement : d'identifier les unités les plus efficientes dans un réseau d'unités comparables maillant un territoire; de détecter les meilleures pratiques et de les généraliser avec des standards très précis; de gérer la temporalité qui est l'élément central de la prestation de service que ce soit un temps actif ou un temps perdu ; de déplacer la demande, d'optimiser les prix, de calibrer et d'optimiser les capacités de production; de mesurer la qualité perçue par le client et d'essayer d'articuler coûts et valeur dans l'offre de service; de mettre sous tension l'organisation par l'usage d'indicateurs articulés dans des relations de cause à effet à l'intérieur d'un tableau de bord stratégique d'ensemble.

Les résultats de trois travaux de recherche consacrés respectivement à l'étude du lien entre modèles d'affaires et contrôle opérationnel dans la restauration commerciale (Goureaux et Meyssonnier, 2011), au contrôle des réseaux de franchise (Goullet et Meyssonnier, 2011) ou à la gestion des temps et des délais dans les services publics de proximité (Meyssonnier et Tahar, 2011) ont été intégrés dans la réflexion.

Les enjeux actuels du contrôle de gestion dans les services nous semblent tourner autour de la normalisation plus ou moins grande des processus productifs (standardisation ou bien empowerment et knowledge management), du développement d'offres globales de service (intégration autour de l'offreur dominant ou coopération entre spécialistes restant autonomes) et de la place croissante de la technologie (qui amène à repenser le rôle du personnel de contact soit par le développement d'un middle office, soit par la généralisation des pratiques de self service). Bien évidemment la forme et les modalités des systèmes de pilotage de la performance dépendront de la nature des services. Certains auteurs (Baranger et alii, 2009) distinguent, en fonction de l'évolutivité des savoirs et de l'intensité de l'aspect relationnel de la prestation de service, des entreprises de service «minimales», «programmées », « servicielles » et «apprenantes ». Pour nous, dans les deux premiers cas, le contrôle de gestion assurant le respect des régularités en matière de comportement et de résultat sera essentiel. Dans les deux derniers, l'autonomie des acteurs du front office et le développement des connaissances des communautés de pratiques constituées par les personnels de contact seront privilégiés. 
Notre contribution à l'étude des spécificités du contrôle de gestion des services s'est faite en s'appuyant sur un recensement des préoccupations propres aux services et une caractérisation de l'instrumentation liée. Certes il est bon de «dépasser l'analyse élémentaire de l'instrumentation de gestion pour étudier sa mise en cohérence » (Aggeri et Labatut, 2010), encore faut-il d'abord connaître et comprendre les pratiques managériales et outils de gestion utilisés dans les services. Ce travail préalable a mis en évidence trois dimensions fondamentales des systèmes de pilotage de la performance dans les services «purs » à la personne : (1) la recherche et la généralisation des meilleures pratiques opérationnelles dans le réseau des unités de terrain, (2) la mise en œuvre d'une batterie de dispositifs assurant l'ajustement permanent aux fluctuations de la demande dans le temps, (3) l'alignement de toutes les composantes de l'organisation vers la valeur créée pour le client. Nous envisageons de développer maintenant une recherche plus fine consacrée au design des systèmes de pilotage de la performance dans les services prenant en compte toute une série de paramètres comme les caractéristiques du service, les attentes de la clientèle, les points d'accès au service, etc. Un tel travail devrait déboucher sur la construction d'une typologie contingente des configurations de références (ou idéaux types au sens de Weber) qui articulent en leur sein de façon cohérente l'usage des divers instruments du contrôle de gestion serviciel.

\section{Bibliographie}

Aggeri, F., Labatut, J. (2010). La gestion au prisme de ses instruments. Une analyse généalogique des approches théoriques fondées sur les instruments de gestion. Finance-Contrôle-Stratégie, 13 (3) : 5-37.

Allain, E. (2010). La modélisation des coûts dans le secteur des services : une lecture au travers de la variable «temps », Thèse de l'université Rennes 1.

Anthony, R. (1965). Management Planning and Control System : A Framework for Analysis, Harvard Business School Press.

Bancel-Charencol, L., Jougleux, M. (19997). Un modèle d'analyse des systèmes de production dans les services. Revue Française de Gestion, 113 : 71-81.

De Bandt, J., Gadrey, J. (1994). Relation de service, marchés de services. CNRS Editions.

Baranger, P., Dang Nguyen, G., Leray, Y., Mével, O. (2009). Le management opérationnel des services. Economica.

Berry, L., Lampo, S. (2000). Teaching an Old Service New Tricks - The Promise of Service Redesign, Journal of Service Research, $3: 265-275$.

Bessire, D. (1998). Logiques d'entreprise et design du contrôle de gestion : une comparaison entre le commerce de detail integer et la banque commerciale. Finance-Contrôle-Stratégie, 1 (4) : 5-37.

Bitner, M. (2000). The Servicescape, in Handbook of Services Marketing and Management, Schwartz et Iacobucci Eds, Sage Publication.

Bitran, G., Ferrer, J.-C., Rocha e Oliveira, P. (2008). OM Forum - Managing Customer Experiences: Perspectives on the Temporal Aspects of Service Encounters, Manufacturing and Service Operations Management, 10 (1) : 61-83.

Bouquin, H. (2006). Le contrôle de gestion. Dunod. 7ème édition.

Broadbent, J., Laughlin, R. (2009). Performance Management Systems: A Conceptual Model, Management Accounting Research, 20 : 283-295.

Capiez, A. (2003). Yield Management, Optimisation du revenu dans les services, Lavoisier.

Chenhall, R. (2003). Management Control Systems Design within its Organizational Context: Findings from Contingency-Based Research and Directions for the Future. Accounting, Organizations and Society, 28 (2-3) : 127-168.

Chiapello, E. (1996). Les typologies des modes de contrôle et leurs facteurs de contingence : un essai d'organisation de la literature. Comptabilité-Contrôle-Audit, 2 (2) : 51-74. 
Collier, D., Meyer, S. (1998). A Service Positioning Matrix, International Journal of Operations and Production Management, 18 (12) :1223-1244.

Crandall, R., Markland, R. (1996). Demand Management - Today's Challenge for Service Industries. Production and Operations Management, 5 (2) : 106-120.

Cugini, A., Caru, A., Zerbini, F. (2007). The Cost of Customer Satisfaction: A Framework for Strategic Cost Management in Service Industries, European Accounting Review, 16 (3) : 499-530.

Deville, A., Leleu, H. (2010). De nouvelles mesures pour comparer la performance opérationnelle et financière des agences bancaires. Comptabilité-Contrôle-Audit, 16 (2) : 97-126.

Djellal, F., Gallouj, C. (2007). L'économie des services. Presses Universitaires de Grenoble.

Ducrocq, C., Gervais, M., Herriau, C. (2001). Le suivi de la qualité et des coûts dans les enterprises de service : une enquête sur les pratiques et les outils employés par les départements de contrôle de gestion. Finance-Contrôle-Stratégie, 4 (3) : 89-122.

Durrande-Moreau, A. (1999). Waiting for Service: Ten Years of Empirical Research, International Journal of Service Industry Management, 10 (2) : 171-189.

Eiglier, P. (2004). Marketing et Stratégie des Services, Economica.

Eiglier, P., Langeard, E. (1987). Servuction : le marketing des services, Mac Graw Hill.

Ferreira, A., Otley. D. (2009). The Design and Use of Performance Management Systems: An Extended Framework for Analysis, Management Accounting Research, 20 : 263-282.

Fitzsimmons, J., Fitzsimmons, M. (2008). Service Management, $6^{\text {ème }}$ édition, McGraw-Hill.

Gadrey, J. (1997). Introduction au dossier consacré aux services. Revue Française de Gestion, nº 113 : 60-61.

Gadrey, J., Zarifian, P. (2001). L'émergence d'un modèle du service: enjeux et réalités. Editions Liaisons.

Gervais, M. (2009). Contrôle de gestion des activités de service, in Encyclopédie de Comptabilité, Contrôle de gestion et Audit, $2^{\text {ème }}$ édition : 547-562.

Gervais, M., Thenet, G. (2004). Comment évaluer la productivité dans les activités de service ?, Comptabilité-Contrôle-Audit, 10 (1) :147-163.

Goudarzi, K., Eiglier, P. (2006). La socialisation organisationnelle du client dans les entreprises de service : concept et dimensions, Recherche et Applications en Marketing, 21 (3) : 65-90.

Goullet C. et Meyssonnier F. (2011). Le contrôle des réseaux de franchise. Comptabilité-ContrôleAudit, avril, à sortir.

Goureaux P. et Meyssonnier, F. (2011), Business Models et contrôle opérationnel dans les services : le cas de la gestion conjointe des coûts, de la qualité et des délais dans la restauration commerciale, Revue Sciences de Gestion, 82 : 41-58.

Edvardsson, B., Gustafsson, A., Roos, I. (2005). Service Portraits in Service Research: A Critical Review. International Journal of Service Industry Management, 16 (1) : 107-121.

Hayes, R., Wheelwright, S. (1979). Linking Manufacturing Process and Product Life Cycles, Harvard Business Review, 57 (1) : 133-140.

Heineke, J., Davis, M. (2007). The Emergence of Service Operations Management as an Academic Discipline. Journal of Operations Management, 25 (2) : 364-374.

Johnston, R., (1995). The Zone of Tolerance: Exploring the Relations between Service Transactions and Satisfaction with the Overall Service, International Journal of Service Industry Management, $6(5): 46-61$.

Jougleux, M. (2006). Enrichir l'approche théorique de la qualité dans les services : qualité du service et qualité de service, Recherche et Applications en Marketing, 21 (3) : 19-46.

Kaplan, R. (2009). Conceptual Foundations of the Balanced Scorecard in Handbook of Management Accounting Research, Chapman, Hopwood et Shields Eds, Elsevier : 1253-1269.

Kaplan R. et Anderson S. (2008). TDABC : La méthode ABC pilotée par les temps. Les Editions d'Organisation.

Kimes, S., Chase, R. (1998). The Strategic Levers of Yield Management, Journal of Service Research, $1: 156-166$.

Klassen, K., Rohleder, T. (2001). Combining Operations and Marketing To Manage Capacity and Demand in Services, The Service Industries Journal, avril :1-30.

Klassen, K., Russel, R., Chrisman, J. (1998). Efficiency and Productivity Measures for High Contact Services, The Service Industries Journal, octobre : 1-18. 
de La Villarmois, O. (1999). Evaluer la performance des réseaux bancaires : la méthode DEA, Décision Marketing, 16 : 39-51.

Lorino, P. (2008). Méthodes de recherche en contrôle de gestion: une approche critique, FinanceContrôle-Stratégie, 11 (numéro spécial, août) :149-175.

Lovelock, C., Gummesson, E (2004). Whither Services Marketing? In Search of a New Paradigm and Fresh Perspectives, Journal of Service Research, 7 (1) : 20-41.

Lovelock, C., Wirtz, J., Lapert, D., Munos, A. (2008). Marketing des Services, Pearson Education.

Mac Nair, C., Polutnik, L., Silvi, R. (2001). Cost Management and Value Creation: The Missing Link, European Accounting Review, 10 (1) : 33-50.

Maister, D. (1997), True Professionalim, The Free Press.

Maister, D. (2001). Practice What you Preach, Free Press.

Malleret, V. (2009). Peut-on gérer le couple coûts-valeur ?, Comptabilité-Contrôle-Audit, 15 (1) : 7 34.

Malmi, T., Brown, D. (2008). Management Control Systems as a Package - Opportunities, Challenges and Research Directions, Management Accounting Research, 19 (4) : 287-300.

Metters, R., Frei, F., Vargas, V. (1999). Measurement of Multiple Sites in Service Firms with Data Envelopment Analysis. Production and Operations Management, 8 (3) : 262-281.

Meyssonnier, F. (2001). Le target Costing : un état de l'art, Finance-Contrôle-Stratégie, 4 (4) : 113 138.

Meyssonnier, F. (2008). L'instrumentation de la gestion du triplet Cô̂t-Qualité-Délai dans les services (de l'analyse des pratiques professionnelles à un modèle explicatif d'ensemble), Congrès des IAE, Lille.

Meyssonnier, F., Tahar, C. (2011). Le contrôle des temps et des délais dans les services publics communaux, Actes du congrès de l'AFC, Montpellier.

Mills, P., Margulies, N. (1980), «Towards a Core Typologiy of Service Organisation », Academy of Management Review, 5 (2) : 255-265.

Morrison-Coulthard, L. (2004). Measuring Service Quality, International Journal of Market Research, 46 (4) : 479-497.

Olivier, R. (1996). Satisfaction: A Behavioral Perspective on the Consumer, Mc Graw Hill.

Orsingher, C. (2006). Le script de service : fondements du concept et applications au marketing des services, Recherche et Applications en Marketing, 21 (3) : 115-128.

Otley, D. (1999). Performance Management: A Framework for Management Control Systems Research, Management Accounting Research, 10 : 363-382.

Parasuraman, A., Zeithaml, V., Berry, L. (1985). A Conceptual Model of Service Quality and its Implications for Future Research, Journal of Marketing, 49 : 41-50.

Roth, A., Menor, L. (2003). Insight into Service Operations Management: A research Agenda, Production and Operations Management, 12 (2) : 1059-1478.

Sampson, S., Froehle, C. (2006). Foundations and Implications of a Proposed Unified Services Theory, Production and Operations Management, 15 (2) : 329-343.

Sasser, W., Olsen, R., Wyckof, D. (1978). Management of Service Operations, Allyn and Bacon.

Shostack, G. (1992). Understanding Services through Blueprinting, in Advances in Services Marketing and Management (Eds, Swartz, R. et al.), JAI Press.

Simons, R. (1995). Levers of Control. Harvard Business School Press.

Van Looy, B., Gemmel, P., Van Dierdonck, P. (2003). Services Management, $2^{\text {ème }}$ édition, Prentice Hall.

Vargo, S., Lusch, R. (2004). The Four Service Marketing Myths: Remnants of a Goods-Based, Manufacturing Model, Journal of Service Research, 6 (4) : 324-335.

Weatherford, L., Bodily, E. (1992). A Taxonomy and Research Overview of Perishable-Asset Revenue Management : Yield Management Overbooking and Pricing, Operations Research, 40 (5) : 831-844.

Zeithaml, V. (1981). «How Consumer Evaluation Processes Differ Between Goods and Services », in Donnely, J., George, W. (Eds) Marketing of Services, American Marketing Association : 186-190.

Zeithaml, V., Parasuraman, A., Berry, L. (1985). Problems and Strategies in Services Marketing. Journal of Marketing, 49 (2) : 33-46. 